\title{
SPECTRAL CONCENTRATION FOR PERTURBED EQUATIONS OF HARMONIC OSCILLATOR TYPE
}

\author{
B. M.BROWN AND M. S. P. EASTHAM
}

\begin{abstract}
Sturm-Liouville potentials of the form $x^{a} f(\epsilon x)$ are considered, where $a>0, f$ decays sufficiently rapidly at infinity, and $\epsilon$ is a small positive parameter. It is shown that there are a finite number $N(\epsilon)$ of spectral concentration points, and computational evidence is given to support the conjecture that $N(\epsilon)$ increases to infinity as $\epsilon$ decreases to zero.
\end{abstract}

\section{Introduction}

A long-standing result of Weyl [9] establishes the discreteness of the spectrum associated with the Sturm-Liouville equation

$$
y^{\prime \prime}(x)+\{\lambda-q(x)\} y(x)=0 \quad(0 \leqslant x<\infty)
$$

when the potential

$$
q(x) \rightarrow \infty \text { as } x \rightarrow \infty .
$$

Here, as usual, $q(x)$ is real-valued and locally integrable in $[0, \infty)$, and there is a boundary condition at $x=0$ which, for convenience, we take to be the Dirichlet condition $y(0)=0$. Thus, subject to condition (2), equation (1) has an increasing sequence of real eigenvalues $\left\{\lambda_{n}\right\}$ with $\lambda_{0}$ finite and $\lambda_{n} \rightarrow \infty$ as $n \rightarrow \infty$ (see [8, Section 5.4] and [9]). Simple examples are the harmonic oscillator (or Hermite) potential $q(x)=x^{2}$, for which $\lambda_{n}=4 n+3$, and the Airy potential $q(x)=x$.

Recently, in the context of their theory of spectral instability and complex resonances, Aslanyan and Davies [1] have drawn attention to the potential

$$
q_{\epsilon}(x)=x^{2} \exp \left(-\epsilon^{2} x^{2}\right)
$$

where $\epsilon(>0)$ is a real parameter. In an obvious sense, $q_{\epsilon}(x)$ approximates to the harmonic oscillator potential $x^{2}$ when $\epsilon$ is small. However, because $q_{\epsilon}$ is $L(0, \infty)$, another result going back to Weyl (see [8, Section 5.4] and [9]) is that, with $q_{\epsilon}$ in place of $q$ in equation (1), the spectrum is continuous in the spectral interval $[0, \infty)$ and there are no positive eigenvalues. The original $\lambda_{n}(=4 n+3)$ are perturbed off the real axis into points known as complex resonances, as discussed in [1] and elsewhere [6, Chapter 16].

In this paper, we show that recent theoretical and computational methods for investigating spectral concentration can be applied to potentials of which equation (3) is an example, and our results indicate the extent to which complex resonances influence the real spectrum for such potentials. To define spectral concentration, we recall that, irrespective of condition (2), the equation (1) has associated with it a non-decreasing spectral function

$$
\rho(\mu) \quad(-\infty<\mu<\infty)
$$


which is unique when equation (1) is in the Weyl limit-point case. The Fourier-transform and Parseval formulae for equation (1) are expressed in terms of $\rho(\mu)$ [8, Chapter 6]. Then, following [2, Section 1], we say that spectral concentration occurs at a point $\mu_{0}$ if

1. $\rho^{\prime}$ exists and is continuous in a neighbourhood of $\mu_{0}$, and

2. $\rho^{\prime}$ has a local maximum at $\mu_{0}$.

For potentials such as (3), it is natural to speculate that spectral concentration occurs near to the unperturbed eigenvalues $\lambda_{n}$ when $\epsilon$ is suitably small (see [7, Section 20.3]). In this paper we show that, for a class of potentials of which equation (3) is an example, spectral concentration occurs near to only a finite number $N(\epsilon)$ of the $\lambda_{n}$. More precisely, we show that there is an interval $(0, M(\epsilon))$ within which spectral concentration points are confined, and we obtain an estimate for the growth of $M(\epsilon)$ as $\epsilon \rightarrow 0$.

In Section 2, we give a formula for $\rho^{\prime}$ which is the basis for both our theoretical and computational methods, and we introduce the class of potentials to be considered, (3) being an example. The main result on $M(\epsilon)$ is then stated as a theorem. In Sections 3 and 4, we present our computational findings on the location of spectral concentration for a number of examples of the type (3). These findings indicate that, as $\epsilon$ decreases to zero, $N(\epsilon)$ is non-decreasing with spectral concentration occurring first in connection with $\lambda_{0}$. It would be interesting to have a theoretical proof of this property, and we return to this matter at the end of the paper.

\section{Localisation of spectral concentration}

We begin by presenting a recent formula for $\rho^{\prime}$ which is valid when the potential $q(x)$ in equation (1) satisfies

$$
q(x) \in L(0, \infty)
$$

and $\mu>0$. We recall that the Dirichlet boundary condition $y(0)=0$ has been imposed. Then, with $\mu=s^{2}$, we have

$$
\pi \rho^{\prime}(\mu)=s \exp \left(-s^{-1} \int_{0}^{\infty} q(x) \sin 2 \theta(x, \mu) d x\right)
$$

(see [3, Section 2], [5]), where $\theta(x, \mu)$, considered as a function of $x$, satisfies the first-order differential equation

$$
\theta^{\prime}(x, \mu)=s-s^{-1} q(x) \sin ^{2} \theta(x, \mu)
$$

and the initial condition $\theta(0, \mu)=0$.

The class of potentials to be considered, which includes that given by equation (3), is

$$
q_{\epsilon}(x)=x^{a} f(\epsilon x),
$$

where $a>0, \epsilon>0$,

$$
t^{a+1} f(t) \in L(0, \infty)
$$

and

$$
f(t) \rightarrow 1 \text { as } t \rightarrow 0 .
$$

Now $q_{\epsilon}$ certainly satisfies condition (4), and hence there is a continuous spectrum for $\mu>0$ with equation (5) valid for the corresponding spectral function $\rho_{\epsilon}(\mu)$. Also, as in the case 
of potential (3), $q_{\epsilon}$ is a perturbation of the potential $x^{a}$, the latter having a discrete spectrum consisting of eigenvalues $\lambda_{n}$.

The condition (8) also implies that $x q_{\epsilon}(x) \in L(0, \infty)$, and our first result is then a simple consequence of [5, Theorem 1].

Property 2.1. There is a number $M(\epsilon)$ depending on $\epsilon$, such that $\rho_{\epsilon}^{\prime \prime}(\mu)>0$ for all $\mu>M(\epsilon)$.

It follows from this property that $\rho_{\epsilon}^{\prime}$ does not have a local maximum in the interval $(M(\epsilon), \infty)$, and hence spectral concentration can occur only in the finite interval $(0, M(\epsilon))$. Information on the nature of $M(\epsilon)$ as $\epsilon \rightarrow 0$ can be obtained from the more detailed results in [5, Sections 3 and 4 ] subject to differentiability conditions on $q_{\epsilon}$. We assume, for example, that $q_{\epsilon}^{\prime \prime}$ exists, so that the theory in [5, Section 4] is applicable. As in [5, Section 4], we require several infinite integrals involving $q_{\epsilon}, q_{\epsilon}^{\prime}$ and $q_{\epsilon}^{\prime \prime}$ as follows: $I, I^{\prime}, I^{\prime \prime}, J^{\prime}, J^{\prime \prime}, K, K^{\prime}, L, L^{\prime}, \bar{L}$ and $H$ are respectively the integrals over $[0, \infty)$ with the integrands

$$
\begin{gathered}
\left|q_{\epsilon}\right|, \quad\left|q_{\epsilon}^{\prime}\right|, \quad\left|q_{\epsilon}^{\prime \prime}\right|, \quad\left|\left(x q_{\epsilon}\right)^{\prime}\right|, \quad\left|\left(x q_{\epsilon}\right)^{\prime \prime}\right|, \\
q_{\epsilon}^{2}, \quad\left|q_{\epsilon} q_{\epsilon}^{\prime}\right|, \quad x q_{\epsilon}^{2}, \quad\left|\left(x q_{\epsilon}^{2}\right)^{\prime}\right|, \quad\left|q_{\epsilon}\left(x q_{\epsilon}\right)^{\prime}\right|, \quad x\left|q_{\epsilon}^{3}\right| .
\end{gathered}
$$

As conditions on $q_{\epsilon}$ (or $f$ ) at $\infty$, these integrals are all taken to be convergent, and the easily verified sufficient conditions are stated in the following theorem. The only comment concerns the condition $a \geqslant 1$, which arises because $q_{\epsilon}^{\prime \prime}(x)$ contains a term $a(a-1) x^{a-2} f(\epsilon x)$, and integrability down to $x=0$ requires $a \geqslant 1$.

Theorem 2.2. Let $a \geqslant 1$ and let $f^{\prime}$ be $A C_{\mathrm{loc}}[0, \infty)$. Suppose that conditions (8) and (9) hold, and suppose that

$$
t^{a+1} f^{\prime}(t) \in L(0, \infty), \quad t^{a+1} f^{\prime \prime}(t) \in L(0, \infty) .
$$

Then there are numbers $v$ and $\delta$, independent of $\epsilon$, such that Property 2.1 holds, with

$$
M(\epsilon)=\left(v \epsilon^{-a-1}+\delta \epsilon\right)^{2} .
$$

Further, $v=\beta^{1 / 3}$ with

$$
\beta=2\left(\int_{0}^{\infty} t^{a}|f(t)| d t\right)\left\{2\left(\int_{0}^{\infty} t^{a}|f(t)| d d t\right)^{2}+\int_{0}^{\infty} t^{2 a+1} f^{2}(t) d t\right\} .
$$

Proof. By [5, equation (4.5)] we have $\rho_{\epsilon}^{\prime \prime}(\mu)>0$ when $\mu>\sigma^{2}$ and $\sigma$ is the unique positive zero of the quartic

$$
s^{4}-A s^{2}-B s-C
$$

with

$$
\begin{aligned}
& A=\frac{1}{2} J^{\prime \prime}, \\
& B=\frac{3}{4}\left(1+\frac{1}{2} \sqrt{3}\right) K+\frac{1}{2} L^{\prime}+\frac{1}{2} \bar{L}+I I^{\prime}+\frac{1}{4} I^{\prime \prime}+2 I\left(\frac{1}{2} J^{\prime \prime}+2 I^{2}+L\right), \\
& C=2 I K+H+\frac{1}{2} K^{\prime}+2 I \bar{L} .
\end{aligned}
$$




\section{Spectral concentration}

In each of the infinite integrals occurring here, we make the change of variable $\epsilon x=t$ to factor out the dependence on $\epsilon$. This gives

$$
\begin{aligned}
& A=\alpha \epsilon^{-a}, \\
& B=\beta \epsilon^{-2 a-3}+\beta_{1} \epsilon^{-2 a-1}+\beta_{2} \epsilon^{-a+1}, \\
& C=\gamma \epsilon^{-3 a-2}+\gamma_{1} \epsilon^{-2 a},
\end{aligned}
$$

where $\alpha, \beta, \beta_{1}, \beta_{2}, \gamma$ and $\gamma_{1}$ are independent of $\epsilon$. In particular, $\beta$ arises from the terms $2 I\left(2 I^{2}+L\right)$ in $B$, and is therefore as stated in equation (12). Then, with

$$
w=\epsilon^{a+1} s
$$

in the quartic (13), it is a question of the unique positive zero $w_{0}$ of the quartic

$$
Q(w) \equiv w^{4}-\alpha \epsilon^{a+2} w^{2}-\left(\beta+\beta_{1} \epsilon^{a+2}+\beta_{2} \epsilon^{2 a+4}\right) w-\left(\gamma \epsilon^{a+2}+\gamma_{1} \epsilon^{2 a+4}\right) .
$$

Now $w_{0}$ cannot in general be expressed in a convenient way in terms of the coefficients in $Q$. We therefore seek to identify an explicit value $w_{1}(>0)$ of $w$ which makes $Q\left(w_{1}\right)>0$. We can then say that $w_{1}>w_{0}\left(=\epsilon^{a+1} \sigma\right)$, and hence Property 2.1 holds with

$$
M(\epsilon)=\left(\epsilon^{-a-1} w_{1}\right)^{2} \text {. }
$$

We consider

$$
w_{1}=\beta^{1 / 3}+\delta \epsilon^{a+2},
$$

where $\delta(>0)$ is to be chosen independent of $\epsilon$. Then, by equation (15),

$$
\begin{aligned}
Q\left(w_{1}\right) / w_{1} & >\beta+3 \beta^{2 / 3} \delta \epsilon^{a+2}+3 \beta^{1 / 3} \delta^{2} \epsilon^{2 a+4} \\
& -\alpha \epsilon^{a+2}\left(\beta^{1 / 3}+\delta \epsilon^{a+2}\right)-\left(\beta+\beta_{1} \epsilon^{a+2}+\beta_{2} \epsilon^{2 a+4}\right) \\
& -\left(\gamma \epsilon^{a+2}+\gamma_{1} \epsilon^{2 a+4}\right) \beta^{-1 / 3} .
\end{aligned}
$$

Grouping together the terms in $\epsilon^{a+2}$ and $\epsilon^{2 a+4}$, we have $Q\left(w_{1}\right)>0$ if

$$
3 \beta^{2 / 3} \delta \geqslant \alpha \beta^{1 / 3}+\beta_{1}+\gamma \beta^{-1 / 3}
$$

and

$$
3 \beta^{1 / 3} \delta^{2} \geqslant \alpha \delta+\beta_{2}+\gamma_{1} \beta^{-1 / 3}
$$

Clearly, $\delta$ can be chosen to satisfy both these inequalities. This proves the theorem, with equation (11) following from equations (16) and (17).

It follows from equation (11) that, as $\epsilon \rightarrow 0$,

$$
M(\epsilon) \sim\left(\nu \epsilon^{-a-1}\right)^{2},
$$

and the question arises as to whether approximation (18) provides a good estimate for the length of an interval within which spectral concentration points must lie. The computational results of Section 4 suggest that approximation (18) is numerically inefficient, as we shall discuss later. However, it is not clear that approximation (18) can be improved in the absence of any method other than the one in [5], on which Theorem 2.2 is based. The reason is that the formulae in [5] contain a term

$$
\exp \left(s^{-1} \int_{0}^{\infty}|q(x)| d x\right)=\exp \left(s^{-1} \epsilon^{-a-1} \int_{0}^{\infty} t^{a}|f(t)| d t\right)
$$




\section{Spectral concentration}

by equation (7). Thus, to avoid exponential domination by this term in the formulae in [5], we must have

$$
\left(s \epsilon^{a+1}\right)^{-1} \leqslant \text { (const.) }
$$

for no spectral concentration. Hence no improvement in the power of $\epsilon$ in approximation (18) is forthcoming.

We end this section with three examples of the type (7) for which $v$ can be calculated in equation (11) and approximation (18). We shall return to these examples when we present our computational findings in Section 4.

Example 2.3. $q(x)=x \exp (-\epsilon x)$.

Here $a=1$ and $f(t)=\exp (-t)$ in equation (12). Hence evaluation of the integrals in equation (12) gives $\beta=19 / 4$. Then, for small $\epsilon$, approximation (18) gives $M(\epsilon) \sim$ $(19 / 4)^{2 / 3} \epsilon^{-4}=(2.826) \epsilon^{-4}$.

Example 2.4. $q(x)=x^{2} \exp \left(-\epsilon^{2} x^{2}\right)$.

Here $a=2$ and $f(t)=\exp \left(-t^{2}\right)$ in equation (12). Evaluation of the integrals in equation (12) gives

$$
\beta=\frac{1}{16} \sqrt{\pi}(\pi+1)=0.459
$$

Thus, for small $\epsilon$, approximation (18) gives $M(\epsilon) \sim(0.595) \epsilon^{-6}$.

Example 2.5. $q(x)=x /\left(1+\epsilon^{4} x^{4}\right)$.

Here $a=1$ and $f(t)=1 /\left(1+t^{4}\right)$ in equation (12). Evaluation of the integrals in equation (12) gives

$$
\beta=\frac{\pi}{16}\left(\pi^{2}+2\right)=2.33
$$

Thus for small $\epsilon$, approximation (18) gives $M(\epsilon) \sim(1.76) \epsilon^{-4}$.

\section{First-order approximation}

In this section and the next, we consider the spectral interval $(0, M(\epsilon))$ within which spectral concentration may occur. The Sturm-Liouville equation (1), with the perturbed potential (7), can be written

$$
y^{\prime \prime}(x)+\left[\lambda-x^{a}-x^{a}\{f(\epsilon x)-1\}\right] y(x)=0 .
$$

Now let us assume that there is a power series expansion

$$
f(t)=\sum_{r=0}^{\infty} a_{r} t^{r} \quad(0 \leqslant t<R)
$$

where $a_{0}=1$ by the property (9). Then a standard method in perturbation theory expresses $y$ and $\lambda$ as formal power series in $\epsilon$. Substitution of these series into equation (21) produces an asymptotic series (as $\epsilon \rightarrow 0$ ):

$$
\lambda_{n}+\sum_{r=1}^{\infty} \lambda_{n}^{(r)} \epsilon^{r}
$$


where

$$
\lambda_{n}^{(1)}=a_{1} \int_{0}^{\infty} x^{a+1} \psi_{n}^{2}(x) d x
$$

and $\psi_{n}$ is a normalised eigenfunction corresponding to the unperturbed eigenvalue $\lambda_{n}$ (see [7, Sections 19.1,19.3 and 20.1]). Truncation of series (23) after a finite number of terms then produces a real spectral point which, for small $\epsilon$, approximates to both a complex resonance [1, Section 5.2] and a presumed real point of spectral concentration [7, p. 260]. We shall consider the first-order approximation

$$
\lambda_{n}+\lambda_{n}^{(1)} \epsilon,
$$

and we note here the following two particular cases.

1. $a=1$. The integral in equation (24) can be evaluated [7, Section 19.18 and equation (20.1.4)] and formula (25) becomes the Titchmarsh approximation

$$
\lambda_{n}+\frac{8}{15} a_{1} \lambda_{n}^{2} \epsilon
$$

2. $a=2$ and $a_{r}=0$ (for $r$ odd) in equation (22). The perturbation parameter is now $\epsilon^{2}$ rather than $\epsilon$, and formula (25) becomes

$$
\lambda_{n}+\lambda_{n}^{(1)} \epsilon^{2}
$$

with $\lambda_{n}=4 n+3$ and, in place of equation (24),

$$
\lambda_{n}^{(1)}=a_{2} \int_{0}^{\infty} x^{4} \psi_{n}^{2}(x) d x .
$$

Again, the integral here can be computed [1, Section 5.2], and we have the AslanyanDavies approximation

$$
4 n+3+\frac{3}{4} a_{2}\left(8 n^{2}+12 n+5\right) \epsilon^{2} .
$$

In the next section, we shall see that both (26) and (27) provide good approximations to actual spectral concentration points.

\section{Computer-assisted investigation}

In [2] and [3], we developed computational procedures to locate spectral concentration on the basis of formulae such as (5) and (6). An example in [2] close to the spirit of this paper is the potential $q(x)=x-\epsilon x^{2}$. We now note that these procedures also apply to the present potentials (7), and we recall the main features as stated in [3, Section 3]. In formulae (5) and (6), the potential $q$ is now $q_{\epsilon}$, and correspondingly $\rho$ and $\theta$ are denoted by $\rho_{\epsilon}$ and $\theta_{\epsilon}$.

We first select a range $[0, M]$ for $\mu$, within which to carry out the computational investigation. This range is chosen to contain a convenient number (say 5 or 10) of the unperturbed eigenvalues $\lambda_{n}$. We also choose a decreasing sequence of values of $\epsilon$ with the aim of exhibiting (a) the approach of the spectral concentration points to the $\lambda_{n}$, and (b) the appearance of new spectral concentration points. For each choice of $\epsilon$, the procedure is as follows.

1. For a given $q_{\epsilon}$, the numerical computation of $\rho_{\epsilon}^{\prime}$ is performed over the range $[0, M]$ at increments of $10^{-2}$. Here we use equation (5) and the numerical solution of equation 
(6) at each mesh point, subject to $\theta_{\epsilon}(0, \mu)=0$. Purpose-written code is then used to examine the output of $\rho_{\epsilon}^{\prime}$ and determine any local maxima at this level of precision.

2. The approximate spectral concentration points found in Stage 1 are used as good starting values to refine the location of the actual points to a higher precision. Thus the process in Stage 1 is repeated now, but only in neighbourhoods of the approximate points, and with a mesh size of $10^{-6}$.

An additional remark on this procedure is occasioned by the observation that spectral concentration becomes intense as $\epsilon$ becomes small, in the sense that the spectral concentration shows itself as a very sharp spike in the graph of $\rho_{\epsilon}^{\prime}$. If the base of this spike is less than the Stage 1 increment of $10^{-2}$, this local maximum may not appear in Stage 1 . We deal with this difficulty by working with a sufficiently fine mesh of values of $\epsilon$, and by tracking the location of each spectral concentration point as $\epsilon$ decreases. If any such point seems to be absent when a certain value of $\epsilon$ is reached, a neighbourhood of the last-known point is searched with the $\mu$-mesh size of $10^{-6}$ to reveal the sharp spike. To illustrate the sharpness of the spike, we give in Table 1 values of $\rho_{\epsilon}^{\prime}\left(\mu_{0} \pm \eta\right)$ and $\rho_{\epsilon}^{\prime}\left(\mu_{0}\right)$ in the case of the first spectral concentration point $\mu_{0}$ for Example 4.1 below (see the second column of Table 2).

Table 1: Intensity of spectral concentration

\begin{tabular}{cccccc}
\hline$\epsilon$ & $\mu_{0}$ & $\eta$ & $\rho_{\epsilon}^{\prime}\left(\mu_{0}-\eta\right)$ & $\rho_{\epsilon}^{\prime}\left(\mu_{0}\right)$ & $\rho_{\epsilon}^{\prime}\left(\mu_{0}+\eta\right)$ \\
\hline 0.18 & 1.77 & 0.01 & 0.53 & 49.81 & 1.17 \\
0.12 & 1.974956 & $10^{-6}$ & 0.2308 & 144.6 & 0.1979 \\
\hline
\end{tabular}

Table 2: Spectral concentration points for Example 4.1

\begin{tabular}{cccccc}
\hline$\epsilon$ & & & & \\
\hline 0.36 & $1.48(1.29)$ & & & & \\
0.18 & $1.77(1.81)$ & & & & \\
0.16 & $1.84(1.87)$ & $2.51(2.66)$ & & & \\
0.13 & $1.94(1.96)$ & $2.79(2.93)$ & & & \\
0.12 & $1.98(1.99)$ & $2.92(3.02)$ & $3.40(3.57)$ & & \\
0.10 & $2.04(2.05)$ & $3.14(3.20)$ & $3.69(3.89)$ & & \\
0.09 & $2.07(2.08)$ & $3.24(3.29)$ & $3.92(4.06)$ & $4.28(4.58)$ & \\
0.08 & $2.10(2.11)$ & $3.34(3.38)$ & $4.13(4.22)$ & $4.58(4.82)$ & $4.985)$ \\
0.05 & $2.19(2.19)$ & $3.63(3.64)$ & $4.68(4.71)$ & $5.50(5.56)$ & $6.16(6.26)$ \\
0.03 & $2.25(2.25)$ & $3.82(3.82)$ & $5.03(5.03)$ & $6.03(6.05)$ & $6.91(6.93)$ \\
\hline
\end{tabular}

In [2] and [3], we noted that spectral concentration is also associated with a certain transitional behaviour of the function $\theta(x, \mu)$ which appears in equation (5). Without repeating the details here, we have found that $\theta_{\epsilon}(x, \mu)$ exhibits a similar behaviour, thus once again providing indirect evidence of spectral concentration. This evidence is particularly useful 
when $\epsilon$ becomes small because then $q\left(=q_{\epsilon}\right)$ in equation (5) becomes more like $x^{a}$ over a long $x$-interval $(0, X)$, and numerical integration in equation (5) becomes computationally less reliable.

In Tables 2, 3 and 4, we return to Examples 2.3, 2.4 and 2.5 respectively (now re-labelled as Examples 4.1, 4.2 and 4.3), and we present our computational findings on the location of spectral concentration points. The figures in brackets are the approximations (26) or (27), as the case may be. The chosen $\mu$-range covers the first five unperturbed eigenvalues $\lambda_{n}$, but there is no difficulty in taking a longer range.

Example 4.1. $q(x)=x \exp (-\epsilon x)$; see Example 2.3 and Tables 1 and 2 .

The first five unperturbed eigenvalues are

$$
2.34, \quad 4.09, \quad 5.52, \quad 6.78, \quad 7.94 \text {. }
$$

We note that the number $N(\epsilon)$ of spectral concentration points increases as $\epsilon$ decreases and, adding to what is shown in the table, we have found that $N(\epsilon)$ is $6,7,8,9$ or 10 when $\epsilon$ is respectively $0.065,0.06,0.055,0.0513$ or 0.051 .

Example 4.2. $q(x)=x^{2} \exp \left(-\epsilon^{2} x^{2}\right)$; see Example 2.4 and Table 3.

As noted in Section 1, the first five unperturbed eigenvalues are 3, 7, 11, 15 and 19. The left-hand column in Table 3 recognises that the parameter in this example is $\epsilon^{2}$ rather than $\epsilon$.

Table 3: Spectral concentration points for Example 4.2

\begin{tabular}{cccccc}
\hline$\epsilon^{2}$ & & & & \\
\hline 0.2 & $2.11(2.25)$ & & & & \\
0.1 & $2.56(2.62)$ & $4.91(5.13)$ & & & \\
0.09 & $2.61(2.66)$ & $4.91(5.31)$ & & & \\
0.05 & $2.80(2.81)$ & $5.90(6.06)$ & $7.96(8.71)$ & & \\
0.04 & $2.84(2.85)$ & $6.16(6.25)$ & $8.70(9.17)$ & $10.65(11.61)$ & \\
0.035 & $2.86(2.86)$ & $6.28(6.34)$ & $9.10(9.40)$ & $11.03(12.03)$ & \\
0.031 & $2.88(2.88)$ & $6.37(6.42)$ & $9.37(9.58)$ & $11.63(12.37)$ & $13.56(14.79)$ \\
0.03 & $2.88(2.89)$ & $6.39(6.44)$ & $9.43(9.62)$ & $11.79(12.46)$ & $13.65(14.93)$ \\
0.02 & $2.92(2.93)$ & $6.61(6.63)$ & $10.01(10.09)$ & $13.10(13.31)$ & $15.80(16.29)$ \\
0.01 & $2.96(2.95)$ & $6.81(6.81)$ & $10.53(10.54)$ & $14.11(14.15)$ & $17.55(17.64)$ \\
0.005 & $2.98(2.98)$ & $6.91(6.91)$ & $10.77(10.77)$ & $14.57(14.58)$ & $18.30(18.32)$ \\
\hline
\end{tabular}

The values of the first spectral concentration point in Table 3 can be compared not only with approximation (27), but also with the complex resonances given in [1, Table $6, \lambda_{1}$ column]. Thus, for example, when $\epsilon^{2}=0.2$, the complex resonance is at $2.03-0.25 i$ compared with our real point 2.11; as $\epsilon$ decreases, the difference between the complex resonance and our real point decreases. 
We also comment on the remark made at the end of [1, Section 5.1] that, for small values of $\epsilon$, there are several resonances very close to the positive real axis but, at a certain point, they turn sharply away into the lower half-plane. This situation manifests itself in Table 3 (and similarly in Tables 2 and 4) in the fact that there are only a finite number of spectral concentration points for a given $\epsilon$ because resonances sufficiently far from the real axis are not producing spectral concentration.

Example 4.3. $q(x)=x /\left(1+\epsilon^{4} x^{4}\right)$; see Example 2.5 and Table 4 .

The first five unperturbed eigenvalues are again those given in (28). Here we give a shortened table which exhibits the same general features as the other two. The parameter values are now expressed in terms of $\epsilon^{4}$.

Table 4: Spectral concentration points for Example 4.3

\begin{tabular}{cllll}
\hline$\epsilon^{4}$ & & & & \\
\hline $10^{-2}$ & $2.10(2.31)$ & & & \\
$5 \times 10^{-3}$ & $2.13(2.33)$ & & & \\
$10^{-3}$ & $2.29(2.34)$ & $3.68(4.08)$ & & \\
$10^{-4}$ & $2.33(2.34)$ & $4.04(4.09)$ & $5.29(5.52)$ & $6.13(6.79)$ \\
\hline
\end{tabular}

The location of spectral concentration points given in Tables 2, 3 and 4 can be compared to the estimates for $M(\epsilon)$ given in Examples 2.3-2.5 by making choices for $\epsilon$. Thus, when $\epsilon=0.1$ in Example 2.3, the approximate value of $M(\epsilon)$ is $2.826 \times 10^{4}$, but the greatest spectral concentration point from Table 2 is only 3.69. Similarly, when $\epsilon=0.2$ in Example 2.4, the corresponding values are 9297 and 10.65 and, when $\epsilon=10^{-3 / 4}$ in Example 2.5, the values are 1760 and 3.68. These figures clearly indicate that the power of $\epsilon$ in approximation (18) is not the best possible and, following our remarks concerning inequality (19), it remains an open question whether some other method would provide a better value of $M(\epsilon)$ than equation (11) as $\epsilon \rightarrow 0$.

We conclude by returning to the question about $N(\epsilon)$ raised at the end of Section 1 . In [2] (see also [4]), we considered potentials which, although having a different nature from (7), share the property that an unperturbed discrete spectrum becomes a continuous spectrum with spectral concentration. Examples in [2] and [4] are $q(x)=x-\epsilon x^{2}$ and $q(x)=-(1+x)^{-1}-\epsilon x$. Both in these examples and in Section 4 of this paper, we find that, as $\epsilon$ decreases, spectral concentration occurs first in connection with the lowest unperturbed eigenvalue $\lambda_{0}$, and then with the higher eigenvalues in turn. Thus $N(\epsilon)$ is nondecreasing as $\epsilon$ decreases to zero, and it would be interesting to know if this is a general property of $N(\epsilon)$, not confined to such examples.

\section{References}

1. A. Aslanyan and E. B. Davies, 'Spectral instability for some Schrödinger operators', Numer. Math. To appear. 76, 76, 81, 81, 83, 84

2. B. M. Brown, M. S. P. Eastham and D. K. R. McCormack, 'Spectral concentration and perturbed discrete spectra', J. Comput. Appl. Math. 86 (1997) 415-425. 77, 81, $81,82,84,84$ 
3. B. M. Brown, M. S. P. Eastham and D. K. R. McCormack, 'Spectral concentration and rapidly decaying potentials', J. Comput. Appl. Math. 81 (1997) 333-348. 77, 81, 81,82

4. M. S. P. Eastham, 'On the location of spectral concentration for perturbed discrete spectra', Mathematika. To appear. 84,84

5. M. S. P. Eastham, 'On the location of spectral concentration for Sturm-Liouville problems with rapidly decaying potential', Mathematika 45 (1998) 23-36. 77, 78, 78, 78, 78, 78, 79, 79, 80

6. P. D. Hislop and I. M. Sigal, Introduction to spectral theory, Appl. Math. Sci. 113 (Springer, 1996). 76

7. E. C. Titchmarsh, Eigenfunction expansions, Part II (Clarendon Press, Oxford, 1958). $77,81,81,81$

8. E. C. Titchmarsh, Eigenfunction expansions, Part I, 2nd edn (Clarendon Press, Oxford, 1962). $76,76,77$

9. H. WEYL, 'Über gewöhnliche Differentialgleichungen mit Singularitäten und die zugehörigen Entwicklungen willkürlicher Funktionen', Math. Ann. 68 (1910) 220-269. $76,76,76$

B. M.Brown malcolmecs.cf.ac.uk

M. S. P. Eastham

Department of Computer Science

Cardiff University

PO Box 916

Cardiff CF24 3XF 